\title{
Clima organizacional y desempeño laboral
}

Mg. Alexandra Del Rocío Aldaz Quinto

alexandra_1976_@hotmail.com

https://orcid.org/0000-0002-6660-0127

Mg. Wendy Yesenia Alvarado Torres

w_alvarado_t@hotmail.com

https://orcid.org/0000-0002-8133-8577

Mg. Nancy Aracely Castro Ramírez

na-chita76@hotmail.com

https://orcid.org/0000-0003-3157-6320

Mg. Catherine Magdalena Fajardo Campaña

cathy_fajardoc@hotmail.com

https://orcid.org/ 0000-0002-9988-3557

\section{RESUMEN}

La investigación tuvo como objetivo determinar la correlación entre el clima organizacional y desempeño laboral de una unidad educativa del cantón Salitre, 2020. La metodología fue de tipo básica, con un enfoque cuantitativo; y diseño no experimental descriptivo de alcance transeccional correlacional, se utilizó el muestreo censal con cuarenta partícipes, la obtención de los datos se dieron a través de una encuesta aplicando un cuestionario para las variables objeto de estudio, las preguntas plasmadas en el instrumento de medición fueron analizadas bajo la rigurosidad de profesionales en el área para paso seguido realizar la prueba de fiabilidad del Alfa de Cronbach con un coeficiente de 0,853 para la variable clima organizacional y 0,827 para la variable desempeño laboral; por ende, los instrumentos empleados son altamente fiables. Como resultados finales se determinó que existe un nivel de significancia inferior de 0.05 , demostrando que el clima organizacional que se percibe en la unidad educativa influyó en el desempeño laboral de los trabajadores y, por consiguiente, existe una marcada correlación entre ambas variables.

Palabras clave: clima organizacional; desempeño laboral; liderazgo y motivación. 


\title{
Organizational climate and work performance
}

\begin{abstract}
The objective of the research was to determine the correlation between the organizational climate and work performance of an educational unit in Salitre, 2020. The methodology was of a basic type, with a quantitative approach; and descriptive non-experimental design of correlational transectional scope, the census sampling with forty participants was used, the data was obtained through a survey applying a questionnaire for the variables under study, the questions expressed in the measurement instrument were analyzed under the rigor of professionals in the area to follow-up to perform the Cronbach's alpha reliability test with a coefficient of 0.853 for the organizational climate variable and 0.827 for the work performance variable; therefore, the instruments used are highly reliable. As final results, it was determined that there is a lower significance level of 0.05 , showing that the organizational climate perceived in the educational unit influenced the work performance of the workers and, therefore, there is a marked correlation between both variables.
\end{abstract}

Keywords: organizational climate; job performance; leadership and motivation.

Artículo recibido: 30 noviembre. 2021 Aceptado para publicación: 29 diciembre 2021 Correspondencia: alexandra_1976_@hotmail.com Conflictos de Interés: Ninguna que declarar 


\section{INTRODUCCIÓN}

A nivel internacional, uno de los mayores desafíos que enfrentan las organizaciones para lograr sus propósitos institucionales es tratar de contar con un grupo de personas comprometidas con seguir los lineamientos que presenta cualquier actividad en cualquier contexto, como ser realistas y competentes en su rol. además de adaptarse a los posibles cambios en función de las singularidades institucionales. Sin embargo, un elemento esencial para que las organizaciones sean prácticas y competentes es explotar sus ventajas humanas, técnicas y económicas, para implantar escenarios agradables de trabajo que permitan a las personas a los empleados dotar a una organización de una ventaja competitiva.

Vera y Suárez (2018) señalan que un buen clima de trabajo dentro de la organización contribuye a una sana competencia entre compañeros, estableciendo un comportamiento maduro entre las partes y facultando a los servidores públicos a ser responsables con la organización a la cual pertenecen; en Ecuador el clima organizacional coincide directamente en el desempeño del personal que para Pilligua y Arteaga (2019) la manera en que un sujeto aprecia su labor, es examinando el contorno humano y físico en el que se desenvuelve el trabajo diario, influyendo directamente sobre su productividad y ese es un tema de suma importancia en la mayoría de las organizaciones que pretenden continuamente mejorar el clima de la organización para lograr incrementar el desempeño laboral.

La unidad educativa Francisco Iñiguez no escapa a este enfoque, aún así, no se realizan diagnósticos del clima organizacional; existiendo información limitada sobre los cambios institucionales necesarios para mejorar e induciendo al descontento por parte del personal en general, que conlleva una reducción del compromiso con las metas de la institución; esto provoca una percepción negativa en la comunidad educativa debido a que no se les brinda el interés adecuado a los servicios solicitados por estos. Por su parte Pereira y Solís (2019) destacan acerca de la importancia en valorar los recursos humanos y medir su desempeño con herramientas que ayuden a estimar su clima organizacional, de manera que se perciban las relaciones establecidas para lograr objetivos comunes.

González y Morales (2020) afirman que actualmente, el análisis del clima organizacional de una entidad es una herramienta estratégica que permite la mejora continua de la entidad. Por lo tanto, al estudiar su comportamiento, se identifican factores importantes 
para mejorar la calidad de vida laboral de los servidores públicos, así como el servicio que brindan. Todo esto se traduce en un buen desempeño laboral, satisfacción, compromiso y eficacia; en consecuencia, de lo antes descrito esta investigación pretende estimar la valoración de los servidores sobre escenarios que aquejan su conveniencia y productividad en el trabajo; asimismo, se aspira que esta sea un referente para el Ministerio de Educación del Ecuador para que analice la significancia de distinguir al personal como elemento fundamental de las unidades educativas del país.

En consecuencia, el objetivo general de la investigación es determinar la correlación entre el clima organizacional y el desempeño laboral del personal de la unidad educativa Francisco Iñiguez Castro del cantón Salitre, 2020; y como hipótesis de investigación comprobar si existe correlación entre el clima organizacional y el desempeño laboral del personal de la unidad educativa Francisco Iñiguez Castro del cantón Salitre, 2020.

\section{MATERIALES Y MÉTODOS}

\section{Enfoque de investigación}

La investigación se efectuó con un enfoque cuantitativo que según Sánchez (2019), son fenómenos que se pueden medir a través de números y que se les pueda dar un tratamiento estadístico.

\section{Tipo de investigación}

Esta investigación fue básica de tipo descriptivo que, según Bentley, Gulbrandsen y Kyvik (2015) una investigación realizada principalmente con el propósito de promover el conocimiento acerca de hechos observables y que no tenga ninguna aplicación o uso particular a la vista se denomina investigación básica; mientras que para Haradhan (2020) la investigación de tipo descriptivo es una forma de estudio que examina detalladamente las características de un fenómeno observado para tener conocimiento de quién, cuándo, dónde, cómo y porqué de la situación.

\section{Diseño de investigación}

El diseño de la investigación fue no experimental y correlacional que según Guerin (2019) la investigación que carece de manipulación de la variable independiente y/o control es no experimentales y para Carrera, Govea, Hurtado y Freire (2019) sí en una investigación se busca determinar el nivel de correspondencia entre dos variables, es un estudio correlacional. 
La metodología fue transeccional que para Martelo, Jaramillo y Ospino (2018) se recopilan los datos en un momento único de tiempo ya que la finalidad es describir las variables en un momento dado.

\section{Población y muestra}

\section{Población}

- La población según Casteel y Bridier (2021) es el total de unidades de análisis a investigar; en este estudio se consideró a los cuarenta servidores públicos de la unidad educativa Francisco Iñiguez de Salitre.

\section{Muestra}

- Como la población de estudio es pequeña se consideró utilizar una muestra censal que para Logan (2018) es un estudio que utiliza todos los elementos de la población para obtener una misma información, conjuntamente se contempló un muestreo probabilístico aleatorio simple que para Otzen y Manterola (2017) este muestreo considera que cada uno de los sujetos que forman parte de la población objetivo tengan iguales oportunidades de ser incluidos en la muestra.

\section{Técnicas e instrumentos de recolección de datos}

La técnica utilizada fue la encuesta que según Phillips (2017) trata acerca de la recopilación de datos primarios acerca de las unidades de análisis, habitualmente mediante la elección de una muestra característica de la población. Como instrumento se aplicó un cuestionario que para Salvador, Marco y Arquero (2021) es un conjunto de preguntas que pueden tener un tipo de respuesta abierta o cerrada; el instrumento tuvo una escala de medición ordinal que según Matas (2018) esta escala contiene un punto medio imparcial, además de puntos a la derecha e izquierda, inicialmente con opciones de desacuerdo y de acuerdo, con valoraciones de contestación seriadas de 1 a 5 .

Por medio del Alfa de Cronbach se midió la fiabilidad de los instrumentos y se obtuvo 0,853 para el cuestionario de la variable de clima organizacional y en desempeño laboral 0,827, calificándose como un instrumento muy confiable. 


\section{RESULTADOS Y DISCUSIÓN}

\section{Tabla 1}

Nivel de clima organizacional y desempeño laboral.

\begin{tabular}{lcccc}
\hline \multirow{2}{*}{ Categorías } & \multicolumn{2}{c}{ Clima organizacional } & \multicolumn{2}{c}{ Desempeño laboral } \\
\cline { 2 - 5 } & $\mathbf{f}$ & $\mathbf{\%}$ & $\mathbf{f}$ & $\mathbf{\%}$ \\
\hline Regular & 1 & 2,5 & 1 & 2,5 \\
Esperado & 24 & 60 & 28 & 70 \\
Destacado & 15 & 37.5 & 11 & 27,5 \\
\hline Total & $\mathbf{4 0}$ & $\mathbf{1 0 0}$ & $\mathbf{4 0}$ & $\mathbf{1 0 0}$ \\
\hline
\end{tabular}

Nota. Cuestionario aplicado a los servidores públicos.

El 2,5\% de los servidores públicos de la unidad educativa Francisco Iñiguez, opinan que el clima organizacional está en un nivel regular debido a la orientación al servicio, mientras que un $60 \%$ lo percibe como esperado por la estructura física de la institución y un 37,5\% como destacado debido al estilo de liderazgo; en concordancia de los resultados que se obtuvieron la teoría del clima organizacional de Halpin y Crofts (1963) aporta indicando que el clima organizacional obedece a la valoración que los individuos tienen acerca de su entorno y que asimismo incide en su desenvolvimiento dentro de la institución.

Simons y otros (2020) creen necesario la aplicación de herramientas que permitan evaluar la percepción que tienen los empleados acerca del clima organizacional, corregir y a la vez juzgar el impacto de iniciativas específicas en concordancia a las necesidades de mejora.

El 2,5\% de los servidores públicos de la unidad educativa Francisco Iñiguez, opinan que el desempeño laboral está en un nivel regular debido a la motivación laboral, mientras que un $70 \%$ lo percibe como esperado por el desempeño individual y un $27,5 \%$ como destacado. En sujeción de los resultados obtenidos Locke afirma que el propósito de lograr un objetivo es una fuente elemental de estimulación en el trabajo para seguir mejorando y tener un buen rol en el ámbito laboral.

Del mismo modo, Agui (2020) indica que el desempeño laboral es proporcional a como se sienta el trabajador en su área de trabajo, ya que no solo se debe menguar las necesidades de estos, sino además ofrecer un buen ambiente organizacional efectuándose actividades eficaces encaminadas a analizar que paralelismo tiene el recurso humano en el progreso y sustentabilidad del éxito institucional. 


\section{Tabla 2}

Categorización de frecuencias de las dimensiones de la variable clima organizacional.

\begin{tabular}{lcccccc}
\hline \multirow{2}{*}{ Categorías } & \multicolumn{9}{c}{ Estilo de liderazgo } & \multicolumn{2}{c}{ Estructura física } & \multicolumn{2}{c}{ Orientación al servicio } \\
\cline { 2 - 7 } & $\mathbf{f}$ & $\mathbf{\%}$ & $\mathbf{f}$ & $\mathbf{\%}$ & $\mathbf{f}$ & $\%$ \\
\hline Regular & 2 & 5 & 1 & 2,5 & 2 & 5 \\
Esperado & 19 & 47,5 & 25 & 62,5 & 21 & 52,5 \\
Destacado & 19 & 47,5 & 14 & 35 & 17 & 42,5 \\
\hline Total & $\mathbf{4 0}$ & $\mathbf{1 0 0}$ & $\mathbf{4 0}$ & $\mathbf{1 0 0}$ & $\mathbf{4 0}$ & $\mathbf{1 0 0}$ \\
\hline
\end{tabular}

Nota. Cuestionario aplicado a los servidores públicos.

El nivel de estilo de liderazgo que aprecia el personal de la unidad educativa Francisco Iñiguez Castro del cantón Salitre es regular en un 5\% y esperado en un 47,5\%, según las contestaciones tomadas en la encuesta; esta observación por parte del personal es debido a que las autoridades sólo se limitan a cumplir órdenes distritales sin tener mayor independencia en la toma de decisiones y al ejecutar labores encomendadas se dificulta por la limitación de los recursos esenciales, además se obtuvo un porcentaje igual para el nivel destacado.

Según Naeem y Azam (2017) infirieron que un liderazgo autocrático es contraproducente para crear un buen clima organizacional y que un liderazgo basado en equipo tiene un mejor resultado cuando de clima organizacional se trata; ya que permite al trabajador conectar el sentido de identidad de sí mismo con los objetivos de la institución y así optimizar su rendimiento.

El nivel de estructura física que considera el personal de la unidad educativa Francisco Iñiguez Castro del cantón Salitre es regular en un 2,5\% y esperado en un $62,5 \%$, considerando las respuestas tomadas de la encuesta; este análisis del personal es dado ya que el colegio cuenta con baños adecuados y espacios acordes a la necesidad del plantel para satisfacción de la comunidad educativa, asimismo se obtuvo un porcentaje para el nivel destacado del $35 \%$.

Esto concuerda con Castro y Morales (2015) quienes aseguran que todo lo relacionado con los espacios físicos de las unidades educativas depende del presupuesto y de la organización por parte de las autoridades y del uso eficaz que le preste tanto el personal administrativo como los docentes por medio de la adecuación de los ambientes educativos. 
El nivel de orientación al servicio considerado por el personal de la unidad educativa Francisco Iñiguez Castro del cantón Salitre es regular en un 5\% y esperado en un 52,5\%, este porcentaje refleja las apreciaciones recolectadas en la encuesta; y se da ya que regularmente se trata de cubrir las necesidades de la comunidad educativa ante sus requerimientos de manera eficaz y a tiempo, también se obtuvo para el nivel destacado el $42,5 \%$.

Esto coincide con Cervantes, Stefanell, Peralta y Salgado (2018) quienes aseveraron que ante la necesidad de alcanzar los objetivos institucionales se presta atención esencial en crear una imagen de eficacia a la hora de gestionar los trámites que la comunidad educativa necesita y por consiguiente las instituciones deben ser conscientes de sus fortalezas y debilidades.

Tabla 3

Categorización de frecuencias de las dimensiones de la variable desempeño laboral.

\begin{tabular}{lcccc}
\hline \multirow{2}{*}{ Categorías } & \multicolumn{2}{c}{ Motivación laboral } & \multicolumn{2}{c}{ Desempeño individual } \\
\cline { 2 - 5 } & $\mathbf{f}$ & $\mathbf{\%}$ & $\mathbf{f}$ & \% \\
\hline Regular & 3 & 7,5 & 2 & 5 \\
Esperado & 22 & 55 & 24 & 60 \\
Destacado & 15 & 37,5 & 14 & 35 \\
\hline \multicolumn{1}{c}{ Total } & $\mathbf{4 0}$ & $\mathbf{1 0 0}$ & $\mathbf{4 0}$ & $\mathbf{1 0 0}$ \\
\hline
\end{tabular}

Nota. Cuestionario aplicado a los servidores públicos.

El nivel de motivación laboral que considera el personal de la unidad educativa Francisco Iñiguez Castro del cantón Salitre es regular en un 7,5\% y esperado en un 55\%, considerando las respuestas obtenidas en la encuesta; estas inferencias otorgadas por el personal son debido a que no existe ningún tipo de compensación por los años de servicio ni se facilitan cursos que ayuden en el perfeccionamiento profesional, por último, en el nivel destacado se reflejó un 37,5\%.

Peña y Villón (2017) confirman que la motivación laboral se puede concebir como consecuencia de la interrelación del sujeto y el estímulo efectuado por la institución con el propósito de instaurar medios que induzcan y estimulen al servidor en alcanzar un propósito. En consecuencia, es el empeño que determina al sujeto por medio del esfuerzo individual a querer alcanzar las metas de la institución adaptado a la necesidad de sosegar sus necesidades personales. 
El nivel de desempeño laboral estimado por el personal de la unidad educativa Francisco Iñiguez Castro del cantón Salitre es regular en un 5\% y esperado en un 60\%, este porcentaje manifiesta las tasaciones reunidas en la encuesta; y se da porque el personal no siente un fuerte vínculo con la institución y muchas de las disposiciones dadas no son precisas, también se obtuvo para el nivel destacado el $35 \%$.

Esto coincide con Enríquez y Calderón (2017) quienes aseguran que entre los factores que más inciden son: el apoyo de las autoridades, la recompensa y la comunicación tanto entre el personal y las autoridades como con los compañeros.

Los datos que se obtuvieron en la encuesta presentaron un comportamiento no normal por consiguiente para verificar el grado de correlación entre las variables se utilizó la medida no paramétrica de coeficiente de correlación de Spearman donde se obtuvo que la variable clima organizacional tiene una correlación significativa (Sig.<0.05) en el desempeño laboral; este resultado permite rechazar la hipótesis nula y se acepta la hipótesis de investigación de que existe correlación entre el Clima Organizacional y el Desempeño Laboral del personal de la unidad educativa Francisco Iñiguez Castro del cantón Salitre.

\section{CONSIDERACIONES FINALES}

1. Se ha confirmado que el clima organizacional guarda una correlación positiva alta en el desempeño laboral del personal de la unidad educativa Francisco Iñiguez Castro del cantón Salitre 2020, ya que se estableció un nivel de significancia menor 0.05, se obtuvo que el clima organizacional se encuentra en un nivel esperado conforme a las encuestas realizadas, también el desempeño laboral se haya en un nivel esperado. Esto permite demostrar que el clima organizacional que se produzca dentro de la institución dispondrá el desempeño laboral de los servidores públicos.

2. Se evidenció que el estilo de liderazgo se ve limitada ante las disposiciones distritales coartando las decisiones de las autoridades en la institución además de que existe restricción de los recursos esenciales como lo son la tecnología, papelería, etc.

3. Se comprobó que la motivación laboral se encuentra en un nivel del 55\% debido a que no existe ningún tipo de compensación por los años de servicio ni se facilitan cursos que ayuden en el perfeccionamiento profesional del personal.

4. Los datos tuvieron una distribución no normal y se empleó para la prueba de hipótesis el coeficiente de correlación de Spearman donde se obtuvo un nivel significancia menor de 0,05 permitiendo aceptar la hipótesis de investigación. 
5. Esta investigación puede utilizarse como referencia para la producción de estudios a futuro fundamentándose en las dimensiones consideradas en esta investigación.

\section{REFERENCIAS BIBLIOGRAFICAS}

Agui, A. (2020). Bienestar y desempeño laboral de los trabajadores públicos de la UNHEVAL, Huánuco, 2019. Gaceta científica, 6(4), 203-212. doi:https://doi.org/10.46794/gacien.6.4.979

Bentley, P., Gulbrandsen, M., \& Kyvik, S. (2015). The relationship between basic and applied researchin universities. High Educ, 70(4), 1-22. doi:http://dx.doi.org/10.1007/s10734-015-9861-2

Carrera, F., Govea, F., Hurtado, G., \& Freire, C. (2019). Estudio Correlacional de Factores como Desempleo e Índices de Delincuencia en Ecuador. Información Tecnológica, 30(3), 287-294. doi:http://dx.doi.org/10.4067/S071807642019000300287.

Casteel, A., \& Bridier, N. (2021). Describing populations and samples in doctoral student research. International Journal of Doctoral Studies, 16(1), 339-362. doi:https://doi.org/10.28945/4766

Castro, M., \& Morales, M. (2015). Los ambientes de aula que promueven el aprendizaje, desde la perspectiva de los niños y niñas escolares. Revista Electrónica Educare, 19(3), 1-32. doi:http://dx.doi.org/10.15359/ree.19-3.11

Cervantes, V., Stefanell, Í., Peralta, P., \& Salgado, R. (2018). Calidad de servicio en una institución de educación superior en la ciudad de Barranquilla. Ciencias Administrativas, $\quad 11(11), \quad 27-41 . \quad$ Obtenido de https://www.redalyc.org/journal/5116/511654337003/511654337003.pdf

Enríquez, M., \& Calderón, J. (2017). El Clima Laboral y su Incidencia en el Desempeño del Personal Docente de una Escuela de Educación Básica en Ecuador. Podium, 1(1), 131-143. Obtenido de https://revistas.uees.edu.ec/index.php/Podium/article/view/77/76

González, D., \& Morales, D. (2020). Clima organizacional y desempeño laboral de los servidores públicos del Servicio de Rentas Internas. Digital Publisher CEIT, 5(5), 79-93. doi: https://doi.org/10.33386/593dp.2020.5.245 
Guerin, B. (2019). The Use of Participatory and Non-Experimental Research Methods in Behavior Analysis. $\quad$ Perspectivas, $\quad 9(9), \quad$ 248-264. doi:http://dx.doi.org/10.18761/PAC.2018.n2.09

Halpin, A. y. (1963). The Organizational Climate and Individual Value Systems upon Job Satisfaction. California, Estados Unidos: Personnel Psychology.

Haradhan, M. (2020). Quantitative Research: A Successful Investigation in Natural and Social Sciences. Munich Personal RePEc Archive, 9(4), 52-79. Obtenido de https://mpra.ub.uni-muenchen.de/105149/1/MPRA_paper_105149.pdf

Logan, J. (2018). Relying on the Census in Urban Social Science. Sage Journal, 17(3), 540-549. doi:https://doi.org/10.1111\%2Fcico.12331

Martelo, R., Jaramillo, J., \& Ospino, M. (2018). roducción científica de docentes universitarios y estrategias para aumentarla mediante series de tiempo y MULTIPOL. Espacios, 39(16), 11-19. Obtenido de https://www.revistaespacios.com/a18v39n16/a18v39n16p11.pdf

Matas, A. (2018). Diseño del formato de escalas tipo Likert: un estado de la cuestión. REDIE, 20(1), 38-47. doi:https://doi.org/10.24320/redie.2018.20.1.1347

Naeem, H., \& Azam, M. (2017). Leadership Styles in the Maintenance Industry of UAE: A Focus Group Analysis. European Scientific Journal, 13(7), 53-67. doi:https://doi.org/10.19044/esj.2017.v13n7p53

Otzen, T., \& Manterola, C. (2017). Técnicas de Muestreo sobre una Población a Estudio. Int. J. Morphol, 35(1), 227-232. Obtenido de https://scielo.conicyt.cl/pdf/ijmorphol/v35n1/art37.pdf

Peña, H., \& Villón, S. (2017). Motivación Laboral. Elemento Fundamental en el Éxito $\begin{array}{lrrr}\text { Organizacional. } & \text { Revista } & \text { Scientific, } & 3(7),\end{array}$ doi:https://doi.org/10.29394/Scientific.issn.2542-2987.2018.3.7.9.177-192

Pereira, A., \& Solís, D. (2019). Factores del clima organizacional (Caso: Instituciones educacionales de San Carlos). Revista Scientific, 4(1), 95-115.

Phillips, A. (2017). Proper Applications for Surveys as a Study Methodology. West JEM, 18(1), 8-11. doi:https://dx.doi.org/10.5811\%2Fwestjem.2016.11.32000

Pilligua, C., \& Flor, A. (2019). El clima laboral como factor clave en el rendimiento productivo de las empresas. estudio caso: Hardepex Cía. Ltda. Cuadernos 
Latinoamericanos de Administración, XV(28), 1-25. Obtenido de https://www.redalyc.org/journal/4096/409659500007/409659500007.pdf

Salvador, J., Marco, G., \& Arquero, R. (2021). Evaluación de la investigación con encuestas en artículos publicados en revistas del área de Biblioteconomía y Documentación. Revista Española de Documentación Científica, 44(2), 1-18. doi:https://doi.org/10.3989/redc.2021.2.1774

Sánchez, F. (2019). Fundamentos epistémicos de la investigación cualitativa y cuantitativa: Consensos y disensos. Revista Digital de Investigación en Docencia Universitaria, 13(1), 102-122. doi:http://dx.doi.org/10.19083/ridu.2019.644

Simons, A., Riedel, N., Toelch, U., Hendriks, B., Müller, S., Liebenau, L., . . Reinhart, M. (2020). Assessing the Organizational Climate for Translational Research with a New Survey Tool. Science and Engineering Ethics, 26(6), 2893-2910. doi:https://dx.doi.org/10.1007\%2Fs11948-020-00234-0

Vera, N., \& Suárez, A. (2018). Incidencia del clima organizacional en el desempeño laboral, el servicio al cliente: Corporación de Telecomunicaciones del Cantón La Libertad. Revista Universidad y Sociedad, 180-186. Obtenido de http://scielo.sld.cu/pdf/rus/v10n1/2218-3620-rus-10-01-180.pdf 